\title{
OPTICAL ASTROMETRY AND THE GLOBAL POSITIONING SYSTEM
}

\author{
P.C. Kammeyer ${ }^{1}$, H.F. Fliegel ${ }^{2}$, R.S. Harrington ${ }^{1}$ \\ ${ }^{1}$ U.S. Naval Observatory, 34th and Massachusetts Ave., NW, Washington, D.C. 20392 \\ 2 Aerospace Corporation, P.O. Box 92957, Los Angeles, California 90009
}

\begin{abstract}
Astrometric accuracies of a few tens of milliarcseconds are expected to be attainable within five years by calibrating astrograph plates with optical observations of Global Positioning System (GPS) satellites against a stellar background. The line of sight from an observer on the Earth's surface to a GPS satellite may be calculated with high accuracy. Motion on each day of the line of sight to the satellite and changes from day to day in the apparent path of the satellite are sufficiently slow to make it possible to reduce atmospheric errors by averaging. Advanced ground-based optical sensors, probably using charge coupled device technology, will be required for GPS optical astrometry.
\end{abstract}

\section{Global Positioning System}

The Global Positioning System (GPS) is designed to provide three-dimensional positions and velocities to navigational users worldwide. Although primarily a military system, many of its products are available to the civilian community. Its "clear access" (C/A) signals give to users who know their height above sea level (e.g. mariners) two-dimensional positions to 100 meters (two sigma). More interesting to scientific users are the precise ephemerides, which are declassified after 48 hours. At present, they are computed by the Defense Mapping Agency (DMA) and distributed by the National Geodetic Survey (NGS). US civilian centers now active in improving GPS ephemeris accuracy include the Center for Space Research of the University of Texas at Austin (Schutz et al., 1989) and the Jet Propulsion Laboratory (Lichten and Border, 1987).

Each GPS satellite is in a near circular orbit of which the period of revolution is 12 sidereal hours. Orbital inclinations are high - 63 to 64 degrees for Block I GPS satellites and 54 to 55 degrees for Block II GPS satellites. When satellite longitudes and latitudes are plotted in cartesian coordinates, the plotted positions nearly form a square wave with amplitude equal to the orbital inclination (Figure 1). Since the distance of a GPS satellite from the Earth's center is approximately four Earth radii, the line of sight from an observer on the Earth's surface to the satellite is within 20 degrees of that point of the celestial sphere with declination equal to satellite latitude and hour angle equal to the difference of observer and satellite longitudes. A GPS satellite is visible when the Sun-satellite-Earth angle is less than 90 degrees and brightest just before eclipse, when that angle is about 13 degrees.

A GPS satellite is maneuvered so that its ground track is nearly fixed in terrestrial latitude and longitude, while its orbital plane moves slowly relative to the stars. Thus, for each point on the Earth, observed values of the satellite's declination and hour angle repeat daily, given values occurring about four minutes earlier each day. Figure 2 shows altitudes at Washington, D.C. of GPS satellites 16, 6, 17, 10, 19, 14, and 21 for late September 1990.

The GPS satellites have several advantages over other satellites for astrometric control. They are approximately magnitude 9 , and satellite motion relative to the stars is of the order of $\mathbf{4 5}$ arc minutes per minute of time. The sky coverage is good -- twenty-one operational satellites are planned, plus three spares, with full global coverage. The path of a GPS satellite scans slowly through a star field over a period of months - - the satellite's ground track is nearly fixed, while the orbital plane moves westward at a rate of 0.03 to 0.04 degree per day relative to the stars. Orbital eccentricity is small, and the apogee advances at a rate of less than 0.02 degree per day. 
The position of the optical center of a GPS satellite relative to an observer on the Earth's surface can currently be calculated to 3- to 5-meter accuracy, relative to an Earth-centered coordinate system oriented by VLBI observations, corresponding to an angular accuracy of 30 to 50 milliarcseconds in the line of sight direction. GPS satellite precise ephemerides now give accuracies of 2 to 4 meters for the center of mass. Errors in the calculated displacement from satellite center of mass to center of light are believed to be at present approximately 1 meter. Over the next five years, significant improvements in orbit determination and in modelling of reflectance are expected for GPS satellites. These should lead to the ability to calculate the line of sight to 5 milliarcseconds.

Stationkeeping maneuvers are occasionally performed. The mean frequency of such maneuvers is one per 1.19 years per satellite. Such maneuvers limit the predictability of satellite motion, but are fully reflected after the fact in the precise ephemerides.

\section{Optical Astrometry Relative to Global Positioning System Lines of Sight}

In order to determine star positions with high accuracy relative to the line of sight to a GPS satellite, one may accumulate into pointlike charge images the photoelectrons arising in a charge-coupled device (CCD) both from photons in an optical image of the satellite and photons in optical images of stars. Pointlike charge images of stars may be formed (Figure 3 ) by placing the CCD in a telescope which follows the stars. Aligning the columns of the CCD with the direction of motion of the image of the satellite and moving charge in half of the CCD from row to row at an appropriate rate causes (Figure 4) the photoelectrons coming from the image of the satellite to be accumulated into a pointlike charge image. Such a method of image motion compensation has been used previously (Monet, 1988) to measure star positions with a fixed telescope. Motions of the telescope are reflected identically in both star and satellite charge images.

An instrument for GPS astrometry might consist of a 0.5 -meter aperture telescope of 4-meter focal length with a 2000 by 2000 pixel CCD, having 20 micron pixels, in its focal plane (Figure 3 ). The time required for the image of a GPS satellite to cross the $C C D$, which corresponds to a 30 by 30 arc minute region of sky, is approximately 40 seconds. A narrow bandpass filter is shown, which reduces the number of photoelectrons to 8000 per pixel for an image of 3 pixel radius, exposed 40 seconds, of a magnitude 10 star (filter transmission is $50 \%$ and CCD efficiency is $40 \%$ ). Such a narrow bandpass makes refraction differences with color insignificant.

The standard deviation of observation errors with the described instrument is expected to be approximately 50 milliarcseconds. This includes a one-fortieth pixel error due to causes within the CCD (Monet, 1988), an error of 25 milliarcseconds due to atmospheric turbulence (Han, 1989), and an error of 25 milliarcseconds ( 2.5 meters) due to inaccuracies in the satellite ephemeris.

Since the path of a GPS satellite scans slowly among the stars, covering approximately one hour of right ascension per year, tens of observed GPS positions can be placed in the region covered by a single astrograph plate. These observations can be used to calibrate the plate to significantly better than the 50 milliarcsecond standard deviation of a single observation.

\section{References}

Han, I.: 1989, Astron. J. 97, 607

Lichten, S.M. and Border, J.S.: 1987, J. Geophys. Res. 92, No. B12, 12751

Monet, D.G.: 1988, in Ann. Rev. Astron. Astrophys. 26, 413

Schutz, B.E., Tapley, B.D., Ho, C.S., Rim, HJ., and Abusali, P.A.M.: 1989, in Proceedings of the Fifth International Geodetic Symposium on Satellite Positioning, Las Cruces, New Mexico, March 1989, Vol. 1, 201 


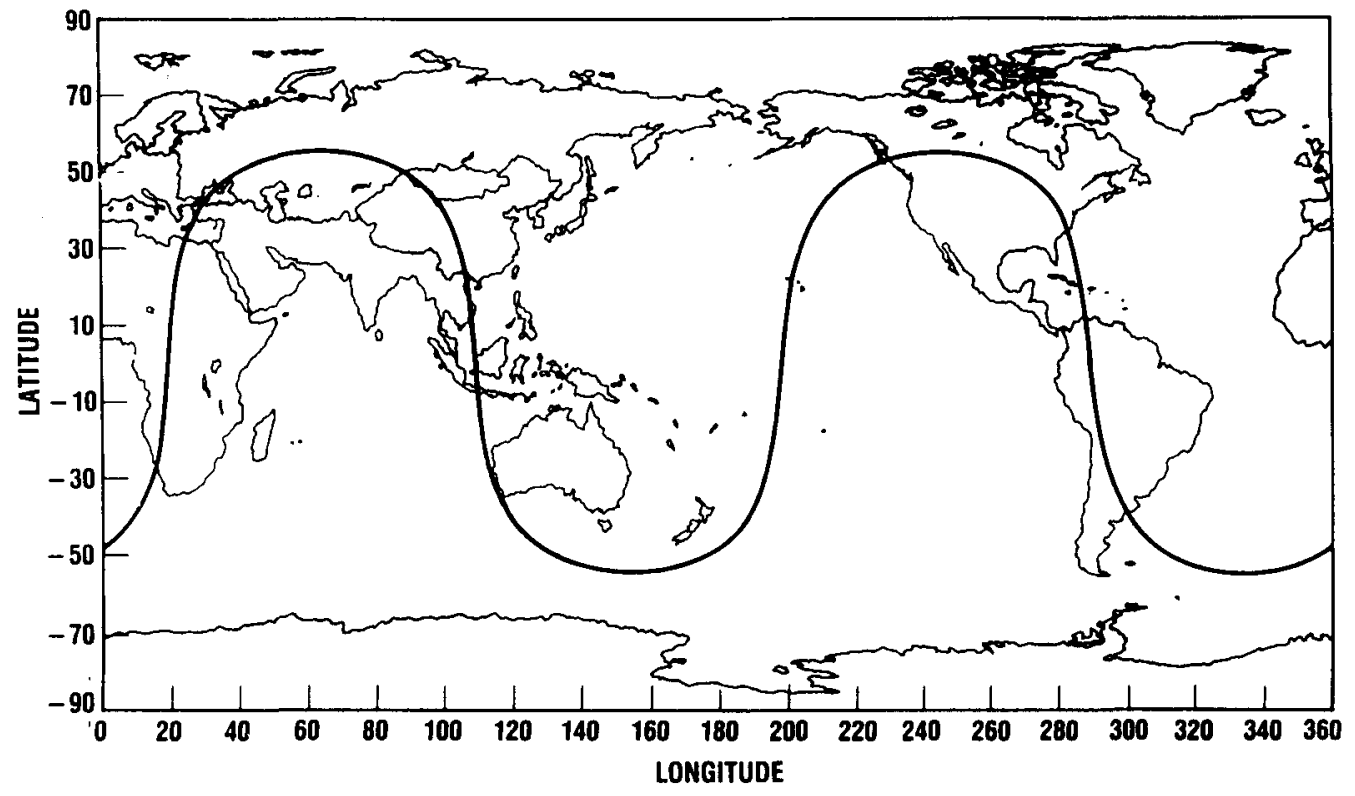

Figure 1. Ground Track of GPS Satellite Number 21 in Late September, 1990

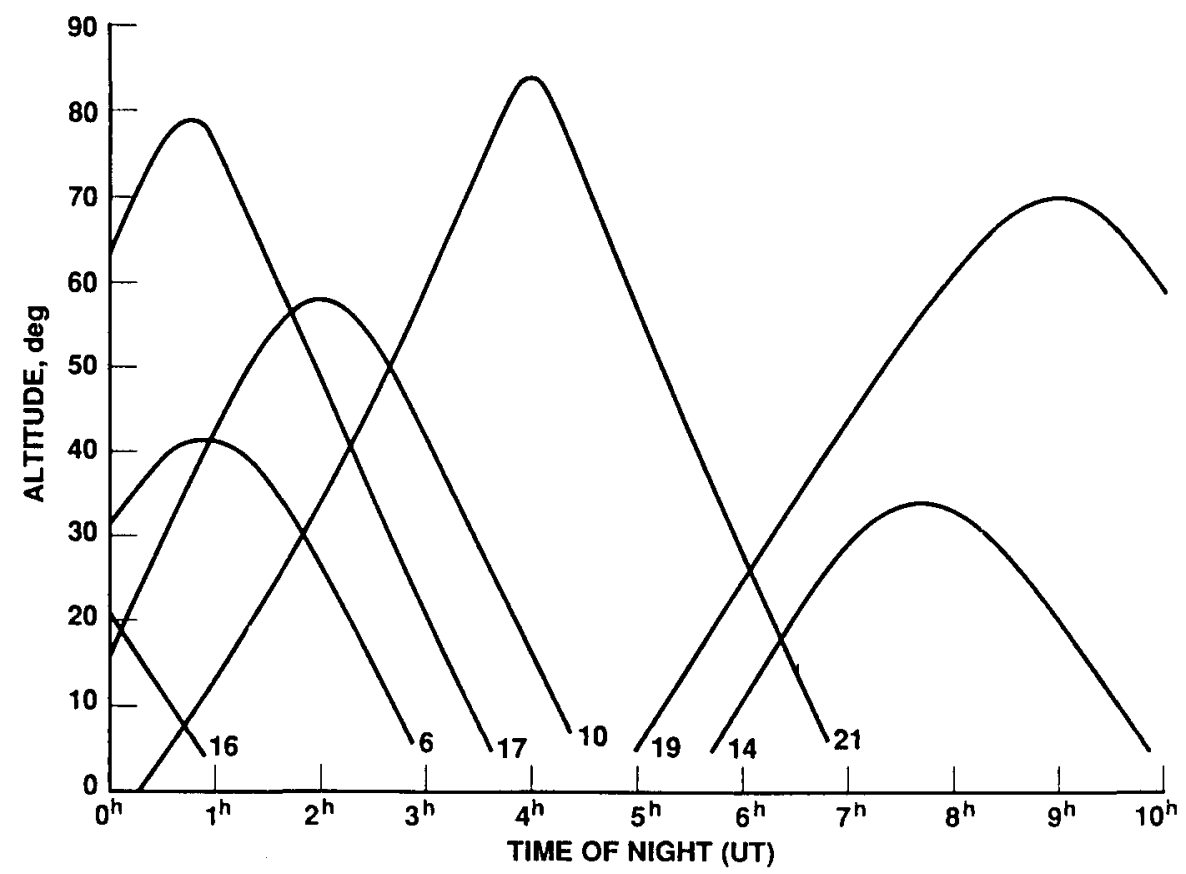

Figure 2. GPS Satellite Altitudes for Washington, D.C. in Late September, 1990 
FROM 0.5-METER E/8 TELESCORE
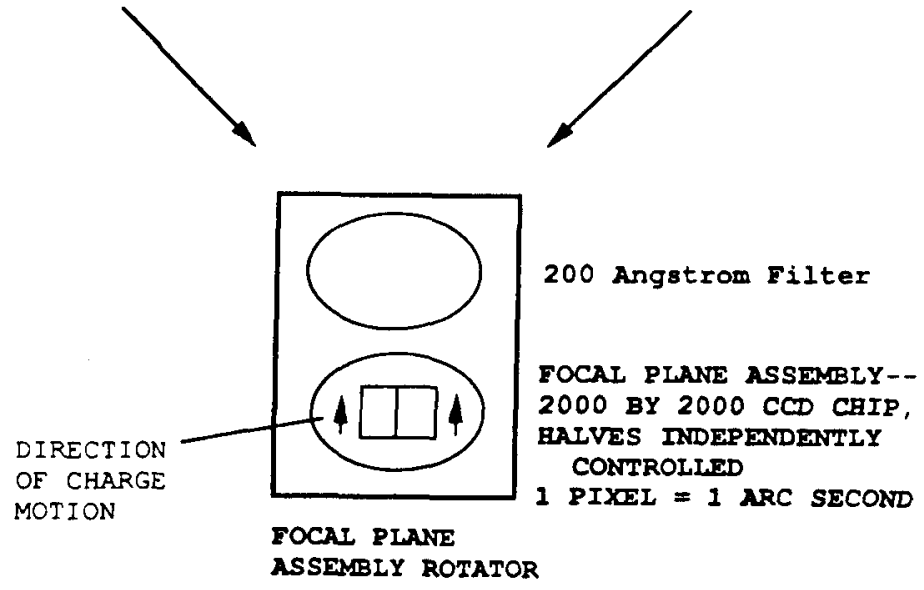

Figure 3. Focal Plane Assembly of GPS Astrometry Telescope

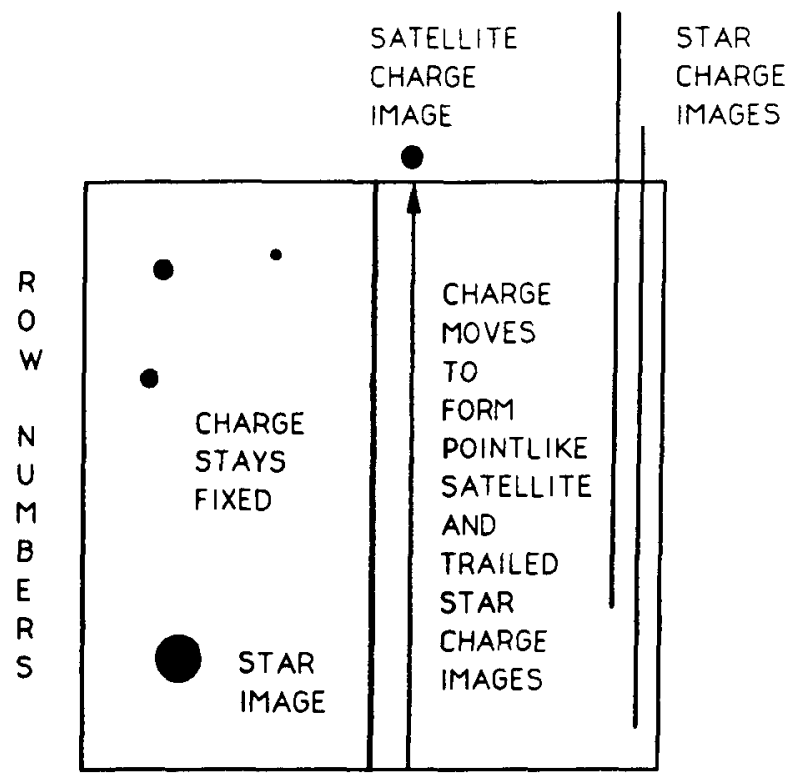

CCD CHIP EDGE WHERE SATELLITE ENTERS

Figure 4. Operation of Focal Plane CCD Chip 